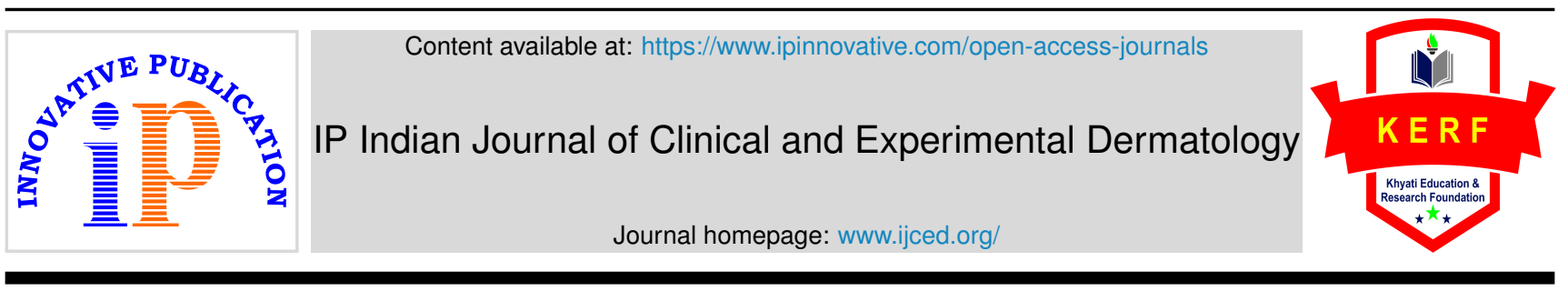

Original Research Article

\title{
A comparative study of efficacy of fractional carbondioxide laser and microneedling fractonal radiofrequency in the treatment of acne scars
}

\author{
K Yashwanth Reddy ${ }^{1, *}$, Ranga Swaroop ${ }^{1}$, Rashmi R Mallaya1, Avik Ghosh ${ }^{1}$, \\ Zigu S Krishn ${ }^{1}$ \\ ${ }^{1}$ Dept. of Dermatology, Adichunchanagiri Institute of Medical Sciences, Mandya, Karnataka, India
}

\section{A R T I C L E I N F O}

\section{Article history:}

Received 29-10-2020

Accepted 25-12-2020

Available online 22-02-2021

\section{Keywords:}

Scabies

Permethrin

Ivermectin

Benzyl Benzoate

Pruritus

\begin{abstract}
A B S T R A C T
Background: Severe acne scars results in permanent scarring and facial disfigurement causing physical and psychosocial distress. Management of acne scars poses a therapeutic challenge for the dermatologists. Ablative lasers do produce significant improvement but associated with post inflammatory hyperpigmentation, prolonged recovery time and scarring. Recently development of fractional carbon dioxide laser and microneedling radiofrequency has proven to be quite effective in the treatment of acne scars. However, till date they have not been compared side by side especially in Indian population. Hence, we intend to conduct the present comparative study.

Objectives: To compare the efficacy of fractional carbon dioxide laser and microneedling fractional radiofrequency in the treatment of facial acne scars.

Materials and Methods: A total of 30 patients (18-40 years) having atrophic scars, attending the out-patient department at the Department Of Dermatology, Venereology and Leprosy, Adichunchanagiri Hospital and Research Centre, B.G. Nagara were alternately allocated into group-A (fractional CO2 group) and group-B (MnRF group). Patients in both the groups received four sequential treatments with an interval of 4 weeks between each session. Clinical assessment of the improvement of atrophic scars was done based on Goodman \& baron grading system at the end of 1 month after the last session, by a side by side comparison of preoperative and post-operative photographs. In addition, patients were asked to provide their opinion on improvement of scars using the patient satisfaction Visual Analogue Score (VAS).

Results: At the end of one month after last session (4 sessions) based on Goodman and Baron's qualitative assessment, $3(20 \%)$ patients showed reduction by 3 grades in group B compared to 2 (13.3\%) patients in group A. $10(66.6 \%)$ patients showed reduction by 2 grades in group B compared to $9(60 \%)$ patients in group A which was found to be statistically not significant ( $\mathrm{p}=0.475)$.

At the end of one month after last session (4 sessions) based on Goodman and Baron's quantitative assessment 3(20\%) patients showed very good reduction in group B compared to 2(13.3\%) in group A $[\mathrm{p}=0.850$ which was statistically not significant $]$.

At the end of 1 month after the last treatment session ( 4 sessions), 33.3\% of patients ( $n=5$ ) in Group B were very satisfied with the treatment as compared to only $13.33 \%$ of patients $(n=2)$ in Group A. The patient satisfaction VAS score after treatment in Group B patients was better when compared to Group A patients [ $\mathrm{p}=0.00086$ which was found to be statistically significant].

The side effects of all 30 patients (100\%) immediately after treatment was erythema and edema which were minimal but $2(13.3 \%)$ patients developed post inflammatory hyperpigmentation in GROUP A whereas none in GROUP B [p=0.0001 which was statistically significant].
\end{abstract}

(C) This is an open access article distributed under the terms of the Creative Commons Attribution License (https://creativecommons.org/licenses/by/4.0/) which permits unrestricted use, distribution, and reproduction in any medium, provided the original author and source are credited.

\footnotetext{
* Corresponding author.

E-mail address: kyashwanthreddy.64@gmail.com (K. Y. Reddy).
}

\section{Introduction}

Acne vulgaris is one of the most common skin diseases affecting more than $85 \%$ of adolescents and adults with 
scarring being commonest sequelae ${ }^{1}$ severe acne can result in permanent scarring and facial disfigurement causing significant physical and psychosocial distress especially in adolescents. $^{2}$

Management of acne scars poses a therapeutic challenge for the dermatologist. Several therapeutic measures like chemical peeling, dermabrasion, subcision, fillers, and punch techniques have been performed with limited efficacy. ${ }^{3}$ Ablative lasers like carbon dioxide ,Erbium, YAG lasers which is based on the principle of selective photothermolysis, can effectively improve facial atrophic acne scarring, but has been hindered in its continued use due to extended post-procedure recovery period and associated morbidity like erythema, post inflammatory hyperpigmentation, hypopigmentation and scarring. ${ }^{4}$ Therefore there is a need for newer therapeutic modalities that are more efficacious, safe with minimal downtime.

The ablative fractional resurfacing creates microscopic treatment zones (MTZ) to stimulate a wound healing response. With this technique, the tissue surrounding each column is spared, ultimately resulting in rapid epidermal regeneration with reduced downtime and adverse reactions compared to treatment with traditional ablative lasers. ${ }^{5}$ This new modality of ablative conventional co2 laser therapy with fractional co2 laser resurfacing was shown to be safe and efficacious in the treatment of acne scars. ${ }^{6}$

A recently developed minimally invasive novel technique is microneedling fractional radiofrequency. Microneedles penetrate into skin with minimal injury to epidermis and once within the dermis, radiofrequency energy is delivered through needles. The heat generated by the resistance offered to passage of radiofrequency energy by the skin causes dermal remodelling, neoelastogenesis and neocollagenogenesis resulting in dermal thickening and skin rejuvenation. ${ }^{7}$ Microneedling fractional radio frequency (MnRF) treatment has demonstrated significant improvement of acne scars, open pores and skin rejuvenation. ${ }^{8}$

Hence, the treatment of acne scars with these two modalities, fractional carbon dioxide laser and microneedling fractional radiofrequency is gaining increased impact showing high efficacy in the treatment of scars. However, till date not been compared side by side especially in Indian population. Through this study, we intend to assess the efficacy of fractional carbon dioxide laser versus that of fractional microneedling radiofrequency in the improvement of facial acne scars.

\section{Materials and Methods}

A total of 30 patients (18-40 years) having atrophic scars, attending the out-patient department at the Department Of Dermatology, Venereology and Leprosy, Adichunchanagiri Hospital and Research Centre, B.G. Nagara were alternately allocated into group-A (fractional $\mathrm{CO} 2$ group) and
group-B (MnRF group). Approval had obtained from institutional ethical committee and a written informed consent was taken from all the patients before enrolling them in the study. A detailed history of the patients as per the prepared questionnaire was taken. A detailed dermatological examination of the face along with photographs of each patient was taken before and after the procedure with emphasis on acne scars.

Patients with history of photosensitive disorders like lupus erythematosus, dermatomyositis, or history of active infections like Herpes type I or II. Pregnant and lactating women and patients with history with scar formation, keloid and vitiligo are excluded from the study.

Patients who were on anti-coagulants, anti-platelet therapy, immunosuppressive drugs, patients with platelet disorders and patients who have received treatment with ablative or non-ablative lasers in the last 12 months were not considered for the study.

\subsection{Group A: Fractional carbon dioxide laser and tratment protocol}

Patients with acne scars had received four sequential fractional carbon dioxide laser (Derma India futura RF30) treatment sessions with an interval of 4 weeks between each session.

The instrument characteristics were as follows:

1. Laser type -ultra pulse, $10600 \mathrm{~nm}, \mathrm{C} 02$ tube

2. Condenser focus $-\mathrm{f}=50 \mathrm{~mm}$

3. Spot size $0.2 \mathrm{~mm}$

4. Pulse frequency $-33.3 \mathrm{~Hz}$

5. Pulse duration $-0.1-10 \mathrm{~ms}$

6. Interval-0.1-2.6mm

7. Repeat $-1-5000 \mathrm{~ms}$

8. Overlap-1-20times

9. Average power-30 $\mathrm{W}$ or 30000 milli-joule per second

10. Scan graphics-square, rectangle, round, triangle, oval and diamond.

11. Dot quanity-400 dots max

12. Scan mode-sequence, random and maximum distance

13. Pulse energy $-10 \mathrm{mj}$ too $30 \mathrm{mj}$ is optional for each dot

Prior to each treatment session, EMLA cream (lignocaine $2.5 \%$ ) was applied to the target regions of acne scars and left for 40mins followed by gentle cleansing.

The procedure area was painted with povidine iodine and cleaned with $70 \%$ isopropyl alcohol as a disinfectant using sterile precautions and eye shields were placed to protect the eyes.

In each session, two passes were administered. The laser procedures were performed with the following settings.

Power: $50 \%$, scanning size: $3 \mathrm{mmx} 3 \mathrm{~mm}$ to $10 \mathrm{mmx} 10 \mathrm{~mm}$ according to the width of the lesion, distance: $1.1 \mathrm{~mm}$, duration $2 \mathrm{~ms}$. 
The first pass was given in sequential mode, targeting only the acne scars and the second pass was given in random mode covering the whole face.

Post procedure, the site was wiped gently with cold water and icepack was applied for 5 minutes to alleviate discomfort and minimize swelling. Patients were advised to apply broad spectrum sunscreen, emollients and to avoid sun exposure for 48 hours post treatment. Any post treatment erythema was treated with topical steroid antibiotic cream provided by investigator and post procedure pain was managed with non-steroidal anti-inflammatory drugs.

\subsection{Group B: Microneedling fractional radiofrequency device and treatment protocol}

Patients with acne scars received four sequential microneedling fractional radiofrequency (DERMA INDIA MR 16-2SB) treatment sessions with an interval of 4 weeks between each session. The energy delivery system consists of a disposable tip with 49 gold-plated micro needle electrodes with a maximum energy output of $50 \mathrm{~W}$. The depth of the needle can be adjusted from a minimum of $0.5 \mathrm{~mm}$ to a maximum of $3.5 \mathrm{~mm}$. When the needle reaches the pre-defined insertion depth the radiofrequency is emitted selectively heating the dermis while sparing the epidermis. The time of needles being out was set as $300 \mathrm{~ms}$ and time difference of radiofrequency and needles being out was set as $2 \mathrm{~ms}$ for each session.

Prior to each treatment session, EMLA cream (lignocaine $2.5 \%$ and prilocaine $2.5 \%$ ) was applied to the targeted regions of acne scars and left for 40minutes followed by gentle cleansing. The procedure area was painted with povidone iodine and cleaned with $70 \%$ isopropyl alcohol as a disinfectant using sterile precautions and eye shield was placed to protect the eyes.

In each session, two passes were administered. The first pass was deep targeting only the scar lesions. Penetration depth was individualized for each scar. Patients with predominantly ice pick scars and mixed scarring were given needle depth of $2.5 \mathrm{~mm}$ and depth was limited to $1.5 \mathrm{~mm}$ on forehead, temple areas and areas with bony prominences. The second pass was superficial targeting the whole face wherein penetration depth of microneedles was limited to $1.5 \mathrm{~mm}$ with minimal or no overlapping. Disposable micro radiofrequency needles were used for every patient in each session.

Post procedure, the sites were wiped gently with cold water and icepack was applied for 5minutes to alleviate discomfort and minimize swelling.

Patients were advised to apply broad spectrum sunscreen, emollients and to avoid sun exposure for 48 hours post treatment. Any post treatment erythema was treated with topical steroid antibiotic cream provided by investigator and post procedure pain was managed with non-steroidal inflammatory drugs for 2-5 days.

\subsection{Clinical Evaluation}

At initial visit (week 0) facial acne scars were graded based on Goodman and Baron's qualitative acne scar grading system. Objectives assessment of physician scores of improvement was determined by Goodman and Baron's qualitative and quantitative acne scar grading system ${ }^{9}$ by a side by side comparison of preoperative and post-operative photographs taken at their first visit and at the end of 1 month after the last session (4 sessions).

In addition patients were asked to provide their opinion about improvement of acne scars using the patients satisfaction visual analogue scale (0-Not satisfied, 1Slightly satisfied, 2-Satisfied, 3-Very satisfied, 4-Extremely satisfied).

Patients were assessed for side effects, such as erythema, edema, pain, bleeding, and post inflammatory hyperpigmentation.

Data was collected and entered in Microsoft excel 2016 and was analysed using SPSS 20.0 Data was presented in the form of percentages, mean and standard deviation. Chi square test and test were used wherever necessary. $\mathrm{p}$ value $<0.05$ was considered as significant at $95 \%$ confidence interval.

\section{Results}

Based on Goodman and baron's qualitative assessment 20\% (3) patients showed reduction by 3 grades in group B compared to $13.33 \%$ (2) patients in group A, $66.66 \%$ (10) patients showed reduction by 2 grades in group B compared to only $60 \%$ (9) patients in group A and $13.3 \%$ (2) patients showed reduction by 1 grade in group B whereas $26.66 \%$ (4) patients showed reduction by grade 1 in group A.

Improvement in acne scar in patients treated with MnRF was slightly more efficacious than patients treated with fractional Co2 laser ( $\mathrm{p}=0.475$ which was not significant).

At the end of one month after last session (4 sessions) based on Goodman and baron's quantitative assessment $20 \%$ showed very good reduction in group B compared to $13.3 \%$ in group A. $26.6 \%$ showed good reduction in both group A and group B, $40 \%$ patients showed moderate reduction in group B whereas $33.3 \%$ showed moderated reduction in group $\mathrm{A}, 13.3 \%$ showed minimal reduction in group B compared to $26.7 \%$ patients in group A $[p=0.850$ which was statistically not significant].

At the end of 1 month after the last treatment session (4 sessions), $33.33 \%$ (5) patients were very satisfied with the treatment in group B compared to $13.33 \%$ (2) patients in Group A. The patient satisfaction VAS score in Group B patients was better when compared to Group A patients $[p=0.000086$ which was found to be statistically significant] The mean Patient Satisfaction VAS Score after four treatment sessions was 2.1 in Group B as compared to 1.8 in Group A ( $<<0.05$ which was statistically significant). 


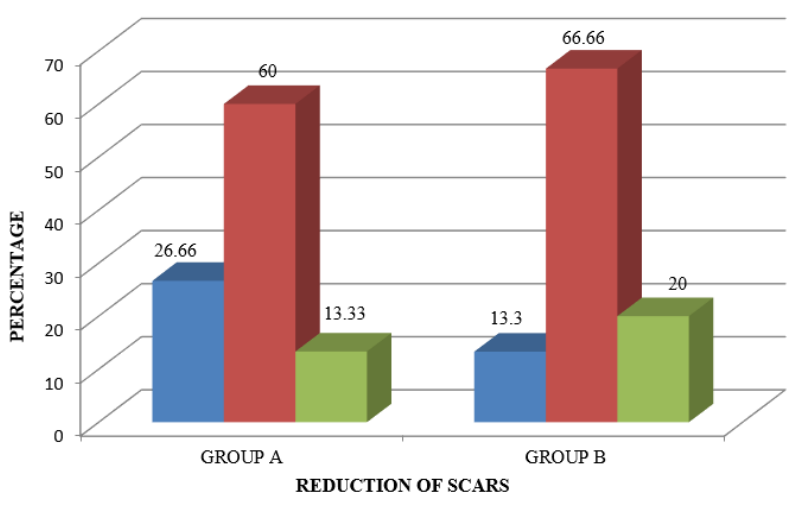

॥EDUCTION BY 1 GRADE $\|$ REDUCTION BY 2 GRADE $\|$ REDUCTION BY 3 GRADE

Graph 1: Graph 00: Physician's assessment based on Goodman and barons Qualitative assessment of post treatment reduction of Scars Comparing Group a and group B

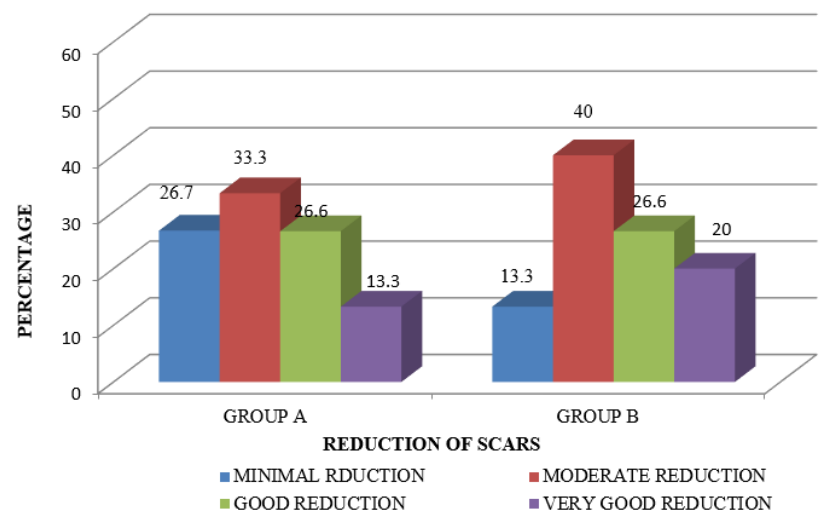

Graph 2: Graph 20: Goodman and baron's quantitative assessment of post treatment reduction of scars, comparing group A and group B.

Patients in both the groups had transient erythema and edema immediately post treatment. $13.3 \%$ (2) patients developed post inflammatory hyperpigmentation in GROUP A whereas none in GROUP B [p=0.0001 which was statistically significant]

\section{Discussion}

Severe acne can result in permanent scarring and facial disfigurement causing significant physical and psychosocial distress. ${ }^{2}$ Atrophic acne scars are more common over the face which results from destruction of collagen following inflammatory acne. ${ }^{10}$

Management of acne scars poses a therapeutic challenge for the dermatologist. Several therapeutic measures like chemical peeling, dermabrasion, subcision, fillers and punch techniques have been performed but with limited

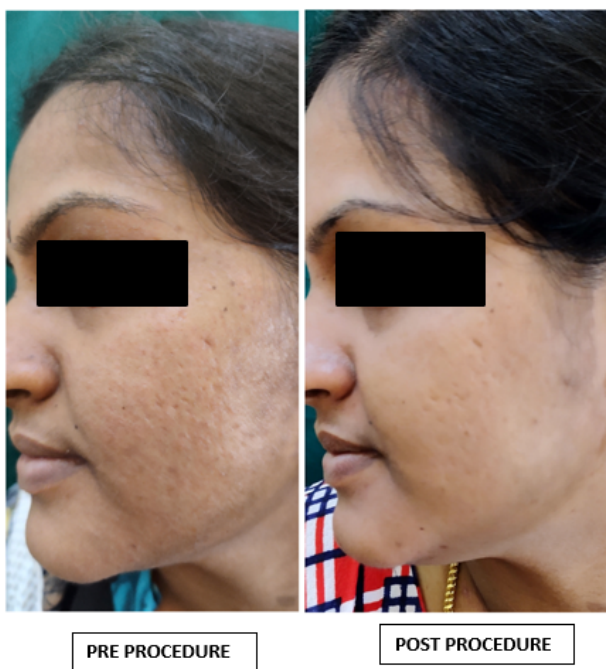

Fig. 1: Pre and post treatment photographof fractional co2 laser patient

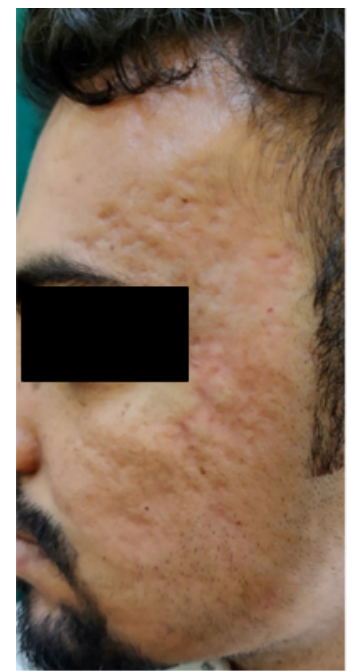

PRE PROCEDURE

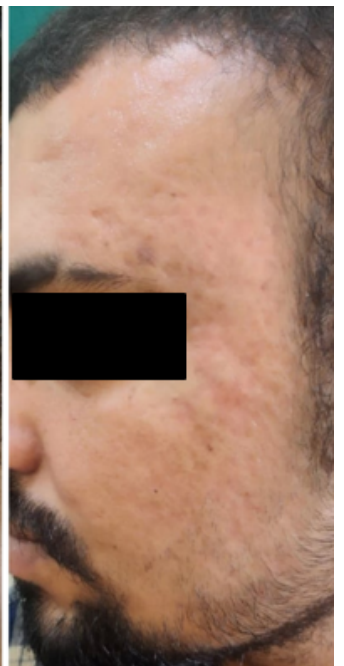

POST PROCEDURE
Fig. 2: Pre and post treatment photographs ofMnRF patient

efficacy. ${ }^{3}$

Ablative lasers like carbon dioxide, Erbium, YAG lasers which is based on the principle of selective photothermolysis can effectively improve facial atrophic acne scarring but has been hindered in its continued use due to extended post-procedure recovery period and associated morbidity like erythema, post inflammatory hyperpigmentation, hypo pigmentation and scarring. ${ }^{4}$

Therefore, there is a need for newer therapeutic modalities that are more efficacious, safe with minimal downtime.

There have been several comparative studies with different modalities like ablative lasers, non-ablative lasers, 
fraction co2 and microneedling radiofrequency, however with extensive review of literature there were no studies comparing the efficacy of fractional carbon dioxide laser and microneedling radiofrequency in the treatment of acne scars.

\subsection{Group A: Fractional carbondioxide laser}

The mean age of patients with acne scars in our study group were 27.40 years which was similar to age distribution (28.2) seen in study done by petrov et $\mathrm{al}^{11}$ and in study done by Majid I et al observed mean age group of 22.1 years. ${ }^{12}$

Acne scar duration of majority of the patients (46.66\%) was found to be between 5 and 10 years which was similar $(46 \%)$ to the study done by Majid I et al. ${ }^{12}$

At the end of one month after the last session based on Goodman and Baron's qualitative assessment, 13.33(2) patients showed reduction by 3 grades, 60\% (9) patients showed reduction of scars by 2 grades, $26.66 \%$ (4) patients showed reduction by 1 grade.

Goodman and Baron's Quantitative assessment revealed, $13.3 \%$ (2) patients had very good reduction, $26.7 \%$ (4) patients had good reduction and $33.3 \%$ (5) patients had moderate reduction and $26.7 \%$ (4) patients showed minimal reduction.

In a similar study done by petrov et al wherein the physician assessment of improvement showed that out of $100 \%$ (40) patients, 33\% (13) patients had excellent improvement, $44 \%$ (17) patients had good improvement, $16 \%$ (6) patients had moderate and 5\% (2) patients had mild improvement. ${ }^{11}$

In study done by QIAN $\mathrm{H}$ et al out of 31 patients receiving three sequential fractional $\mathrm{co} 2$ treatment sessions over a 6 month period, $12.9 \%$ (4) patients showed excellent improvement, $25.8 \%$ (8) patients showed good improvement, $41.9 \%$ (13) patients showed fair improvement and $19.4 \%$ (6) patients showed poor improvement. ${ }^{4}$

Majid I et al observed that out of $100 \%$ (25) patients treated, $60 \%$ (15) patients showed excellent improvement, $24 \%$ (6) patients showed good improvement and 16\%(4) patients showed no improvement. $8 \%$ (2 patients) developed post inflammatory hyperpigmentation. Majority of the patients $76 \%$ (19) patients were highly satisfied with the treatment and $24 \%$ (6) patients were not satisfied. ${ }^{12}$

In study done by Ochi $\mathrm{H}$ et al out of 107 patients treated for acne scarring, out of which $6.4 \%$ (7) patients developed post inflammatory hyperpigmentation which is similar to the present study. ${ }^{13}$

Hedelund et al., conducted a randomised control trial of fractional co2 laser resurfacing for atrophic acne scars, 13 patients with moderate to severe acne scars treated 3 times at 4 to 5 week intervals. Improvement of scar texture and scar atrophy of 1.6 and 1.7, respectively, were found on 10 point scales and only minor adverse effects were reported. ${ }^{5}$
Chapas et al treated 13 patients with moderate to severe acne scars 2 to 3 times at 1 to 2 months interval with fractional co2 laser. Significant improvement of at least 25 to $50 \%$ on a quartile scale were found with no serious adverse effects. ${ }^{14}$

Differences in laser settings, number of patients, number of treatments, treatment intervals, and the degree of acne scar severity and duration made it difficult to directly compare the results from previous study.

At the end of 1 month after last treatment session (4 sessions), out of the 15 patients, $33.33 \%$ (5) patients were very satisfied with the treatment, 7 patients $46.44 \%$ (7) patients were satisfied, and $20 \%$ (3) patients were slightly satisfied with the treatment. In a study done by Majid I et al majority of the patients, $76 \%$ (19) patients were highly satisfied with the treatment.

All patients experienced transient oedema and erythema immediately after the treatment. $13.33 \%$ (2) patients developed post inflammatory hyperpigmentation which resolved thereafter, which was in accordance with Majid I et al. In study done by Ochi $\mathrm{H}$ et al out of 107 patients treated for acne scarring, 6.4\% (7) patients developed post inflammatory hyperpigmentation.

\subsection{Group B: Microneedling radiofrequency}

The mean age of patients with acne scars in our study group were 25.86 years. In study done by Gold et al the mean age group was 35.7 years. ${ }^{15}$ and Y.Harth et al observed mean age of 32.6 years. ${ }^{16}$

Acne scar duration of majority of the patients (46.66\%) was found to be between 5 and 10 years.

At the end of one month after last session, based on Goodman and Baron's qualitative assessment, 20\% (3) patients showed reduction by 3 grades, $66.6 \%$ (10) patients showed reduction by 2 grades and $13.3 \%$ (2) patients showed reduction by 1 grade. Our study results were in concordance with Chandrashekar BS et al, where in $80.64 \%$ of patients showed reduction by 2 grades and $19.35 \%$ showed reduction by 1 grade. ${ }^{17}$

Goodman and Baron's quantitative analysis at the end of one month after last session revealed that $20 \%$ (3) patient had very good reduction, $26.7 \%$ (4) patients had good reduction, $40 \%$ (6) patients had moderate reduction and $13.3 \%$ (2) patients had minimal reduction. The findings were similar to a study done by Chandrashekar BS et al, wherein, $3 \%$ showed very good improvement, $9 \%$ had good improvement, $58 \%$ had moderate improvement and $29 \%$ had minimal improvement. ${ }^{17}$

Another study done by Harth Y et al out of 20 patients, $25 \%$ (5) patients experienced very good to excellent improvement, $30 \%$ (6) patients experienced good and $45 \%$ (9) patients had mild improvement. ${ }^{16}$

Gold et al. conducted a study where in 13 patients with mild to moderate acne scars were treated with bipolar 
fractional radiofrequency and concluded that fractional bipolar radiofrequency is safe and an effective treatment for acne scars. ${ }^{15}$

Cho et al, evaluated efficacy of fractional radiofrequency in treatment of 30 patients with mild to moderate acne scars and large facial pores. The grade of acne scars and investigator global assessment of large pores improved in more than $70 \%$ of the patients. ${ }^{8}$

At the end of 1 month after last treatment session (4 sessions), out of the 15 patients, $33.33 \%$ (5) patients were very satisfied with the treatment, $46.44 \%$ (7) patients were satisfied, and $20 \%$ (3) patients were slightly satisfied with the treatment. Gold et al observed that $65-92 \%$ of patients were satisfied with the results. In a study done by Cho et al, $40 \%$ (12) patients were very satisfied, $46.7 \%$ (14) patients were satisfied and $10 \%$ (3) patients were slightly satisfied.

All patients experienced transient oedema and erythema immediately after the treatment.

In study done by Chandrasekhar et al the MnRF treatment was well tolerated with transient side effects like mild edema, post inflammatory hyperpigmentation and track marks of the devices. ${ }^{17}$

In study done by Ramesh et al observed transient treatment site edema, burning sensation (1 hour), mild scaling and crusting. ${ }^{18}$

\subsection{Comparison of Goodman and baron's qualitative assessment in group $A$ and group $B$}

At the end of one month after last session (4 sessions), $20 \%$ (3) patients showed reduction by 3 grades in group B compared to $13.3 \%$ (2) patients in group A. $66.6 \%$ (10) patients showed reduction by 2 grades in group B compared to $60 \%$ (9) patients in group A which was found to be statistically not significant $(\mathrm{p}=0.475)$.

However there were no similar studies available to compare our study observations.

\subsection{Comparison of patients satisfaction score in group $A$ and group $B$}

At the end of 1 month after the last treatment session (4 sessions), $33.33 \%$ (5) patients were very satisfied with the treatment in group B compared to $13.33 \%$ (2) patients in Group A. The patient satisfaction VAS score in Group B patients was better when compared to Group A patients $[p=0.000086$ which was found to be statistically significant] The mean Patient Satisfaction VAS Score after four treatment sessions was 2.1 in Group B as compared to 1.8 in Group A ( $<<0.05$ which was statistically significant).

\subsection{Comparison of side effects seen in group A and group $b$ patients}

Patients in both the groups had transient erythema and edema immediately post treatment. $13.3 \%$ (2) patients developed post inflammatory hyperpigmentation in GROUP A whereas none in GROUP B [p=0.0001 which was statistically significant].

\section{Source of Funding}

No financial support was received for the work within this manuscript.

\section{Conflict of Interest}

The authors declare they have no conflict of interest.

\section{References}

1. James WD. Clinical practice. Acne. N Engl J Med. 2005;352:146372.

2. Tan JK. Psychosocial impact of acne vulgaris: evaluating the evidence Skin Ther Lett. 2004;9:1-3.

3. Gozali MV, Zhou B, Luo D. Effective Treatments of Atrophic Acne Scars. J Clin Aesthet Dermatol. 2015;8(5):33-40.

4. Qian H, Lu Z, Ding H, Yan S, Xiang L, Gold MH, et al. Treatment of acne scarring with fractional CO2laser. J Cosmet Laser Ther. 2012;87(6):162-5. 101:103 $100 / 74 / 641 / 220126996 / 9$

5. Hedelund L, Haak CS, Togsverd-Bo K, Bogh MK, Bjerring P, Haedersdal M, et al. Fractional $\mathrm{CO} 2$ laser resurfacing for atrophic acne scars: A randomized controlled trial with blinded response evaluation. Lasers Surg Med. 2012;44(6):447-52. 10:-101002//sm.22048

6. Huang L. A new modality for fractional $\mathrm{CO} 2$ laser resurfacing for acne scars in Asians. Lasers Med Sci. 2013;28:627-32.

7. Hruza G, Taub AF, Collier SL, Mulholland SR. Skin rejuvenation and wrinkle reduction using a fractional radiofrequency system. J Drugs Dermatol. 2009;8:259-65.

8. Cho SI, Chung BY, Choi MG, Baek JH, Cho HJ, Park CW, et al Evaluation of the Clinical Efficacy of Fractional Radiofrequency Microneedle Treatment in Acne Scars and Large Facial Pores. Dermatol Surg. 2012;p. 1-8.

9. Goodman GJ, Baron JA. Postacne Scarring: A Qualitative Global Scarring Grading System. Dermatol Surg. 2006;32(12):1458-66. doi:10.1111/.1524-4725.2006.32354.x.

10. Holland DB, Jeremy AHT, Roberts SG, Seukeran DC, Layton AM, Cunliffe WJ, et al. Inflammation in acne scarring: a comparison of the responses in lesions from patients prone and not prone to scar. $\quad B r$ J Dermatol . 2004;150(1):72-81. do1:10.1II1/.1365ए133.200405/49x.

11. Petrov A, Pljakovska V. Fractional Carbon Dioxide Laser in Treatment of Acne Scars. Open Access Maced J Med Sci. 2015;4(1):38-42. doi:10.3889/oamjms.2016.004

12. Majid I, Imran S. Efficacy and safety of fractional CO2laser resurfacing in non-hypertrophic traumatic and burn scars. J Cutan Aesth Surg. 2015;8(3):159-64. 101:10.4103/0974-20/1-16/276.

13. Ochi H, Tan L, Tan WP, Goh CL. Treatment of Facial Acne Scarring With Fractional Carbon Dioxide Laser in Asians, a Retrospective Analysis of Efficacy and Complications. Dermatol Surg. 2017;43(9):1137-43. 10:-10.1097/dss.0000000000001219

14. Chapas AM, Brightman L, Sukal S. successful treatments of acneiform scarring with $\mathrm{co} 2$ ablative fractional resurfacing. Lasers Surg Med. 2008;40:381-387.

15. Gold MH, Biron JA. Treatment of acne scars by fractional bipolar radiofrequency energy. J Cosmetic Laser Ther. 2012;87(6):172-8.

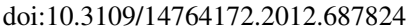

16. Harth Y, Elman M, Ackerman E, Frank I. Depressed Acne Scars-Effective, Minimal Downtime Treatment with a Novel Smooth Motion Non-Insulated Microneedle Radiofrequency Technology. J Cosmetics, Dermatol Sci Appl. 2014;04(03):21218. do1:10.4236/jcdsa.2014.43029 
17. Chandrashekar BS, Sriram R, Mysore R, Bhaskar S, Shetty A. Evaluation of microneedling fractional radiofrequency device for treatment of acne scars. J Cutan Aesthet Surg. 2014;7(2):93-7. 101:10.4103/0974-2077/138328

18. Ramesh M, Gopal M, Kumar S, Talwar A. Novel technology in treatment of acne scars: The matrix tunable radiofrequency technology. J Cutan Aesthet Surg. 2010;214:46-51.

\section{Author biography}

K Yashwanth Reddy, Junior Resident

Ranga Swaroop, Professor and HOD
Rashmi R Mallaya, Junior Resident

Avik Ghosh, Senior Resident

Zigu S Krishn, Junior Resident

Cite this article: Reddy KY, Swaroop R, Mallaya RR, Ghosh A, Krishn ZS. A comparative study of efficacy of fractional carbondioxide laser and microneedling fractonal radiofrequency in the treatment of acne scars. IP Indian J Clin Exp Dermatol 2021;7(1):47-53. 